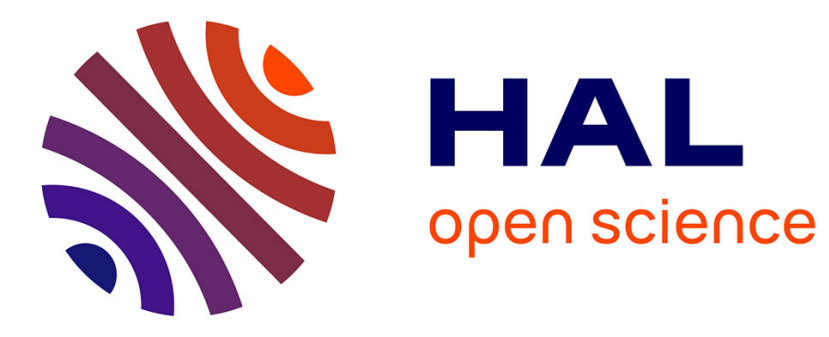

\title{
Towards Perceptual Quality Evaluation of Dynamic Meshes
}

\author{
Fakhri Torkhani, Kai Wang, Annick Montanvert
}

\section{To cite this version:}

Fakhri Torkhani, Kai Wang, Annick Montanvert. Towards Perceptual Quality Evaluation of Dynamic Meshes. APGV 2011 - 8th Symposium on Applied Perception in Graphics and Visualization, Aug 2011, Toulouse, France. pp.116. hal-00628503

\section{HAL Id: hal-00628503 https://hal.science/hal-00628503}

Submitted on 3 Oct 2011

HAL is a multi-disciplinary open access archive for the deposit and dissemination of scientific research documents, whether they are published or not. The documents may come from teaching and research institutions in France or abroad, or from public or private research centers.
L'archive ouverte pluridisciplinaire HAL, est destinée au dépôt et à la diffusion de documents scientifiques de niveau recherche, publiés ou non, émanant des établissements d'enseignement et de recherche français ou étrangers, des laboratoires publics ou privés. 


\title{
Towards Perceptual Quality Evaluation of Dynamic Meshes
}

\author{
Fakhri Torkhani*
}

Kai Wang*

Gipsa-lab, CNRS UMR 5216, Grenoble, France

Annick Montanvert*

\section{Motivation and Objective}

In practical applications, it is common that a 3D mesh undergoes some lossy operations (e.g. simplification, watermarking, compression, noise contamination, etc.). Since the end users of 3D meshes are often human beings, it is thus important to derive metrics that can faithfully assess the perceptual distortions induced by these operations. The derived metrics can be used, for instance, to benchmark a family of geometry processing algorithms, or to guide the design of new algorithms. Like in the case of image quality assessment, metrics based on mesh geometric distances (e.g. Hausdorff distance and root mean squared error) cannot correctly predict the visual quality degradation. Recently, several perceptuallymotivated metrics have been proposed (e.g. mesh structural distortion measure and roughness-based measures) [Lavoué and Corsini 2010]. Those perceptual metrics work well on static meshes, but are less efficient on dynamic meshes because they may mistakenly evaluate "natural" deformations as of rather low perceptual quality (c.f. Section 3). Based on the fact that surface movements in a dynamic mesh sequence are often defined as quasi-isometric deformations (especially in the case of human body and animals animations), we propose in this poster a perceptually-driven mesh quality metric that is capable of distinguishing quasi-isometric deformations from the actual visually unpleasant distortions. To the best of our knowledge, such a metric does not exist in the literature.

\section{Our Method}

There are two main ideas behind our method: (1) constructing the metric by using geometric quantities that are invariant to isometric deformations, namely the Laplace-Beltrami operator and the Gaussian curvature; (2) simulating the characteristics of the human visual system (HVS) in mesh frequency domain.

The first step is to carry out eigendecomposition of the mesh Laplace-Beltrami operator. The obtained eigenstructure (eigenvectors and eigenvalues) constitutes an isometric-invariant mesh spectral analysis tool [Lévy and Zhang 2009]. We then project the mesh's Gaussian curvature onto the eigenvectors, so as to obtain an invariant mesh spectrum. Gaussian curvature has been used also because of its strong connection to the mesh surface normal directions which are important parameters to most of the mesh rendering algorithms. Next, the spectrum is divided into intervals of one $o c$ tave, which simulates the bandpass channel decomposition of HVS. We define geometric contrast as the total energy of the spectrum in each sub-band. This contrast describes to some extent the average mesh irregularity in a given frequency sub-band, considering that Gaussian curvature is a second-order differential quantity which measures the difference between a vertex and its neighbors. In order to compare two meshes, we first compute the difference between their respective contrasts in each sub-band and then combine the differences together. Another important HVS property, the contrast sensitivity function (CSF), has been utilized to deduce the weighting factors for this error pooling stage. Finally, a psychometric function converts the sum of weighted contrast differences $\Delta C$ to a perceptual quality metric $Q$ with values equal to 0 for identical meshes and 1 for visually very different ones.

*e-mail: first.lastname@gipsa-lab.grenoble-inp.fr

Copyright is held by the author / owner(s)

APGV 2011, Toulouse, France, August 27 - 28, 2011.

ISBN 978-1-4503-0889-2/11/0008

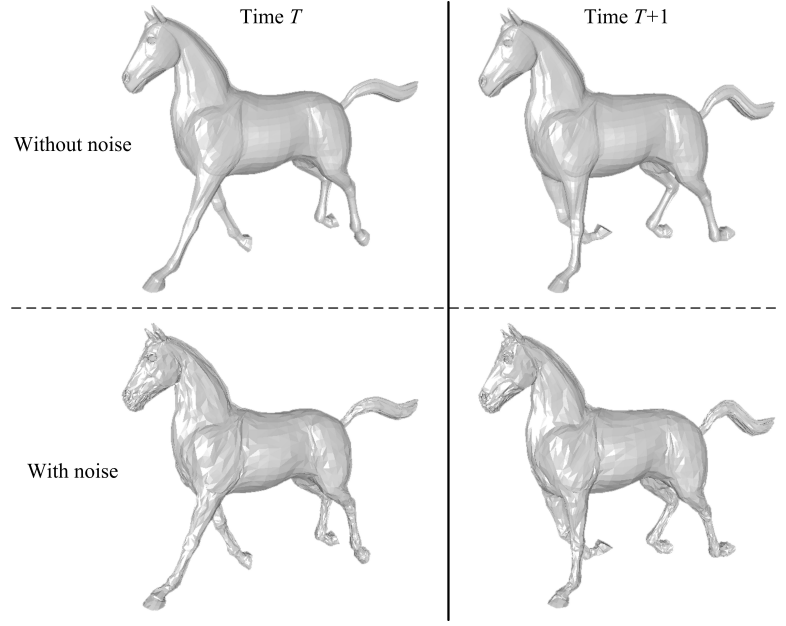

Figure 1: Some Horse models used in our test.

\begin{tabular}{|c|cc|c|c|}
\hline Type of deformation & $\Delta C$ & $Q$ & $M S D M$ & $H_{d}$ \\
\hline \hline low noise & 1.9080 & 0.1036 & 0.2229 & 0.0006 \\
\hline medium noise & 3.6322 & 0.5291 & 0.4372 & 0.0019 \\
\hline high noise & 6.8425 & 0.9970 & 0.5534 & 0.0033 \\
\hline quasi-isometric movement & 1.8195 & 0.0891 & 0.4380 & 0.1530 \\
\hline movement + low noise & 2.0758 & 0.1267 & 0.4998 & 0.1527 \\
\hline movement + medium noise & 3.5824 & 0.5132 & 0.5772 & 0.1521 \\
\hline movement + high noise & 6.6650 & 0.9954 & 0.6327 & 0.1514 \\
\hline
\end{tabular}

Table 1: Some quality/distance evaluation results on Horse, the reference model being the first mesh in the sequence.

\section{Results and Discussion}

We have tested our method on both synthetic and realistic dynamic mesh sequences. For each mesh $\mathcal{M}_{T}$ in a sequence, we measured the distances between this reference model and the following ones: distorted versions of $\mathcal{M}_{T}$ by low/medium/high-amplitude uniform noises, the mesh $\mathcal{M}_{T+1}$ of the next frame in the sequence, and the distorted versions of $\mathcal{M}_{T+1}$ by the above noises. Some dynamic Horse models (8431 vertices, sequence available at http://wwwrech.telecom-lille1.eu/3ddynamicrep/) are shown in Figure 1, and the corresponding results of our quality metric are presented in Table 1, with comparisons to mesh structural distortion measure $(M S D M)$ and Hausdorff distance $\left(H_{d}\right)$. The proposed metric is experimentally more efficient than $M S D M$ and $H_{d}$. Our metric considers a nature quasi-isometric deformation as of very high quality $(Q \approx 0.1)$, and its value increases as the noise amplitude increases. On the contrary, the natural deformation between $\mathcal{M}_{T}$ and $\mathcal{M}_{T+1}$ results in rather big distances between them in terms of $M S D M$ and $H_{d}$. It is worth mentioning that the results of our metric remain stable under a given type of deformation throughout the mesh sequence, which demonstrates its robustness. In the future, we would like to integrate more HVS properties into the metric and use it to guide practical dynamic mesh processing algorithms.

\section{References}

LAVOuÉ, G., AND CORSINI, M. 2010. A comparison of perceptually-based metrics for objective evaluation of geometry processing. IEEE Trans. on Multimedia 12, 7 , 636-649.

LÉvy, B., AND Zhang, H. 2009. Spectral mesh processing. In Proc. of SIGGRAPH Asia Course Notes. 